\title{
Estudio sobre la efectividad de las ortesis plantares en las patologías más frecuentes del pie
}

\author{
Study of the effectiveness of foot orthoses in frequent foot pathologies \\ Isabel María Arias Martín' ${ }^{1}$ Amanda Páez Tudela1', Yolanda Molina Salas² y Pedro V. Munuera Martínez \\ ${ }^{1}$ Departamento de Podología, Universidad de Sevilla, España. ${ }^{2}$ Departamento de Enfermería, Universidad de Murcia, España
}

Palabras clave:

Pie plano, pie valgo, pie cavo, pie varo, tratamiento, ortesis plantares, efectividad, patología del pie.

\section{Resumen}

Introducción: El objetivo del estudio es comprobar la efectividad de las ortesis plantares prescritas en el Área Clínica de Podología de la Universidad de Sevilla para las patologías más frecuentes en el pie.

Pacientes y métodos: Se ha realizado una recopilación de los datos que aparecían en historias clínicas de pacientes atendidos en el Servicio de Ortopodología y Biomecánica de este centro. Se estimó cuáles fueron las patologías más frecuentemente diagnosticadas y cuáles las ortesis más frecuentemente prescritas. La asociación entre la patología tratada, el tipo de ortesis plantar prescrita y otras variables recopiladas se calculó mediante la Odds Ratio (OR). También se comprobó el nivel de mejoría que los pacientes manifestaron en las revisiones posteriores a la entrega del tratamiento.

Resultados: Las patologías diagnosticadas con mayor prevalencia en este servicio fueron las siguientes: el pie plano-valgo, el pie valgo, el pie cavo-valgo, el pie cavo y el pie varo. Además, se puede confirmar que el uso de un soporte plantar a medida produjo una mejora significativa de la sintomatología que originan estas alteraciones en los pacientes que la padecen.

Conclusiones: Como conclusiones, destacar que el tipo de ortesis plantar más usada para el tratamiento de todas ellas fue la estratificada, que el polipropileno fue el material elegido con mayor frecuencia como cuerpo de las ortesis, y que el uso de una cubierta amortiguadora y de un posteado interno o externo de talón fueron los elementos más comúnmente utilizados en dichos tratamientos.

Keywords:

Flatfoot, pes valgus, pes cavus, talipes varus, treatment, foot orthoses, effectiveness, foot pathology.

\section{Abstract}

Introduction: This study aims at checking the effectiveness of the foot orthotics used in the Podiatry Clinical Area of the University of Seville to treat the most frequent foot pathologies.

Patients and methods: It has made a data collection from the medical histories of treated patients by the Orthopaedics and Biomechanics Service of this Centre. The most frequently diagnosed pathologies, and the most frequently foot orthoses employed, were estimated. The association between the alterations treated, the type of orthotic prescribed and other variables recorded in the study, was calculated by means of the Odds Ratio. Also, the level of improvement reported by the patients after the application of the orthotic treatment was reported.

Results: The most prevalent foot diseases diagnoses were the flat-valgus foot, the valgus foot, the cavus-valgus foot, the cavus foot and the varus foot. Moreover, can also said that the use of a custom-made foot orthoses significantly improve the symptoms of these disorders.

Conclusions: The stratified foot insole was the most used for the treatment of these pathologies. The polypropylene was the most chosen material for made the shell of orthoses, and the most common elements used were a shock absorber top cover and a medial or lateral external heel post. 


\section{INTRODUCCIÓN}

La Ortopodología se basa principalmente en el estudio de la biomecánica humana para el tratamiento de las alteraciones funcionales del miembro inferior desde el pie 1 . También trata las patologías propias de este y las que repercuten directamente en él.

La prescripción y realización de ortesis plantares es una de las grandes herramientas terapéuticas de las que dispone la Podología. Sin embargo, según nuestro conocimiento, aún no hay establecido ningún protocolo de actuación ortésica que indique las mejores opciones de tratamiento posibles según la patología existente.

La gama de materiales y elementos que pueden usarse es muy amplia, y son muchas las combinaciones que pueden realizarse. Hay varios factores que influyen en esta decisión, como son el objetivo terapéutico marcado, las características del paciente (peso, edad, actividad) y las propiedades físicas de los propios materiales ${ }^{1,2}$, que le aportan una gran versatilidad. No obstante, son pocos los estudios que evidencian la utilidad de unos u otros en función del diagnóstico establecido, siendo complejo instaurar un criterio de elección, que en general es muy diverso y se basa principalmente en la experiencia y preferencias de los profesionales de Podología.

La ausencia de una guía que marque las pautas a seguir en la elección de un tratamiento según la patología presente, no solo supone un problema a nivel de la práctica clínica sino también en el ámbito legal, ya que deja desprotegido al Podólogo/a ante una situación judicial.

El objetivo del trabajo es estudiar qué tipo de tratamiento ortésico es más efectivo en las patologías diagnosticadas con mayor prevalencia en el Servicio de Ortopodología y Biomecánica del Área Clínica de Podología (ACP) de la Universidad de Sevilla, al ser este nuestro lugar de estudio. Con ello se pretende establecer un criterio de elección en función del éxito y fracaso obtenidos.

\section{PACIENTES Y MÉTODOS}

Diseño

Se llevó a cabo un estudio observacional transversal.

\section{Emplazamiento y participantes}

Mediante un muestreo aleatorio simple, a partir de la base de datos de las historias clínicas de los pacientes atendidos en el Servicio de Ortopodología y Biomecánica del ACP de la Universidad de Sevilla, se seleccionaron 582 historias de un universo muestral de 2000 pacientes atendidos durante dos años. El cálculo del tamaño muestral se realizó con la herramienta online para determinación de tamaños muestrales desarrollada por López-Calviño y cols. (disponible en https://www.fisterra.com/formacion/metodologia-investigacion/determinacion-tamano-muestral/\#23922). Mediante esta herramienta se obtuvo que para una población total de 2000 individuos, un nivel de confianza del $95 \%$, una precisión prevista del $5 \%$, y un valor de proporción del $50 \%$ (peor de los escenarios posibles), el tamaño muestral mínimo necesario era de 322 participantes.

\section{Instrumento y procedimiento para la recogida de datos}

Para la recogida de la información se elaboró un cuestionario donde se recogieron las principales variables del estudio y se codificaron para su posterior análisis estadístico. La variable principal estudiada fue el tipo de ortesis plantar prescrita según las principales patologías del pie. Otras variables que se analizaron fueron los posibles factores asociados a la prescripción de ese tipo de tratamiento corrector.

Las variables recopiladas fueron:

- Número de historia clínica del paciente.

- Sexo.

- Edad.

- Tipo de ortesis plantar realizada en el pie izquierdo y derecho: monolaminar, estratificada o prótesis.

- Materiales usados en cada ortesis:

- Tipo de material o materiales usados en el refuerzo y grosor.

- Tipo de material usado en la cubierta/alargo/forro (si es que presentaban), dureza y grosor.

- Material o materiales usados en el relleno (si es que presentaban), dureza y grosor.

- Uso o no de otros elementos y cuáles:

- Descarga metatarsal.

- Apoyo de primer radio.

- Cuña rotadora externa.

- Talonera.

- Elevación de talón.

- Posteado interno de talón.

- Posteado interno de talón y arco longitudinal interno.

- Posteado externo de talón y arco longitudinal externo.

- Posteado completo de talón.

- Ángulo de antepié varo.

- Ángulo de antepié valgo.

- Alza.

- Cresta subdigital.

- Elemento de amortiguación en antepié.

- Cut-out.

- Motivo de consulta.

- Si realiza alguna actividad física o deportiva, cuál o cuáles y cuántas horas o veces a la semana.

- Profesión.

- Diagnóstico/s.

- Tratamiento complementario: uso o no de otro tratamiento diferente al uso de una ortesis plantar, cuál o cuáles.

- Revisión: si acude o no a revisión. 
- Mejoría: si presenta o no mejoría, y en cuánto tiempo se ha producido.

- Modificaciones: si se realizan o no posteriormente, qué tipo o tipos y cuándo.

- Observaciones: donde se anotaba principalmente si padecía algún tipo de patología previa o antecedente de interés ortopodológico.

De la muestra de 582 pacientes, se dieron como válidas las que permitieron completar la ficha de recogida de datos, siendo motivo de exclusión del estudio las restantes, exceptuando aquellas en las que no se especificaba el grosor y/o dureza del material usado para el forro, cubierta o alargo, el peso y talla, o en las que el paciente no había acudido a revisión. Finalmente, se seleccionaron 330 historias que cumplían los criterios de inclusión. Para aquellos casos en los que no había recogidos datos de peso, talla o resultado del tratamiento, se intentó recopilar esa información mediante encuesta telefónica a posteriori. La revisión de las historias clínicas fue realizada siempre por dos investigadoras simultáneamente, debiendo estar de acuerdo ambas en la selección de la historia clínica y la cumplimentación de la información en la hoja de recogida de datos. Si en algún caso había discrepancia entre las dos investigadoras, se consultaba a un tercer investigador cuyo criterio era discernidor.

\section{Consideraciones éticas}

El estudio fue aprobado por la Dirección del ACP de la Universidad de Sevilla. Todas las historias consultadas fueron revisadas exclusivamente por personal autorizado perteneciente al estudio. Para salvaguardar el compromiso de confidencialidad de los datos adquiridos, los nombres de los participantes fueron sustituidos por números, a efectos de la presentación y discusión de los resultados. Las normas sobre confidencialidad se aplicaron, no solo a los datos almacenados en soporte informático, sino también a aquellos que se encontraban en papel u otro tipo de soporte.

Las normas de confidencialidad siguieron, en todo caso, lo establecido en la Ley Orgánica 15/99 de 13/12/1999, de Protección de Datos de Carácter personal.

\section{Análisis estadístico}

Las variables cualitativas se expresaron en forma de frecuencias y porcentajes. Para las variables cuantitativas se calculó la media y desviación estándar (DE) y, en el caso de que la distribución fuera asimétrica, la mediana y la amplitud intercuartílica $\left(P_{25}, P_{75}\right)$. La comparación de proporciones se realizó mediante la prueba de Chi cuadrado $\left(X^{2}\right)$ y, para la comparación de variables numéricas entre dos grupos, se ha utilizado el test de la prueba de t de Student, una vez cumplidos los requisitos de aleatoriedad, normalidad e igualdad de varianza, usando como medida del efecto la diferencia de medias con su intervalos de confianza (IC $95 \%$ ). Para estudiar asociación entre las variables se calculó la Odds Ratio (OR) con sus correspondientes intervalos de confianza (IC) $95 \%$, la prueba de significación de estas razones se ha calculado con la prueba de Mantel-Haenszel. Los datos se analizaron con el paquete estadístico SPSS en su versión 21.0 para Windows. En el análisis estadístico se consideró un nivel de significación del $5 \%(p \leq 0.05)$.

\section{RESULTADOS}

\section{Resultados generales}

Finalmente se seleccionaron 330 pacientes, el $53.3 \%$ $(n=176)$ eran mujeres, y el resto hombres. La media de edad global de los pacientes fue de 36 años ( $D E=20.7$ ); en hombres fue de 32.7 años ( $D E=20.8$ ) y en las mujeres de 38.9 años (DE = 20.3), $[T=2.7, p=0.007]$. El 69.4\% $(n=229)$ de los pacientes se concentran en una franja de edad entre los 16 y los 65 años.

El $66.4 \%(n=219)$ de los pacientes acudió a consulta por presentar algún tipo de dolor, siendo el localizado en el antepié el más prevalente con el $15.8 \%(n=52)$ de los motivos de consulta. El $9.1 \%(n=30)$ de los pacientes fueron derivados al Servicio de Ortopodología del ACP por otro profesional.

El $88.8 \%(n=293)$ de los pacientes presentaban la misma patología en ambos pies. Las más prevalentes fueron (Figura 1) el pie plano-valgo $16 \%(n=47)$, el pie valgo $15 \%$ $(n=44)$, el pie cavo-valgo $13.7 \%(n=40)$, el pie cavo $10.2 \%$ $(n=30)$, y el pie varo $6.8 \%(n=20)$. Por lo tanto, centraremos los resultados del estudio en estas.

Presentaron un diagnóstico secundario el $28.8 \%(n=95)$ de los pacientes, siendo la insuficiencia del primer radio la que se presentó en mayor proporción (2.7\%), comportándose de igual manera en ambos pies.

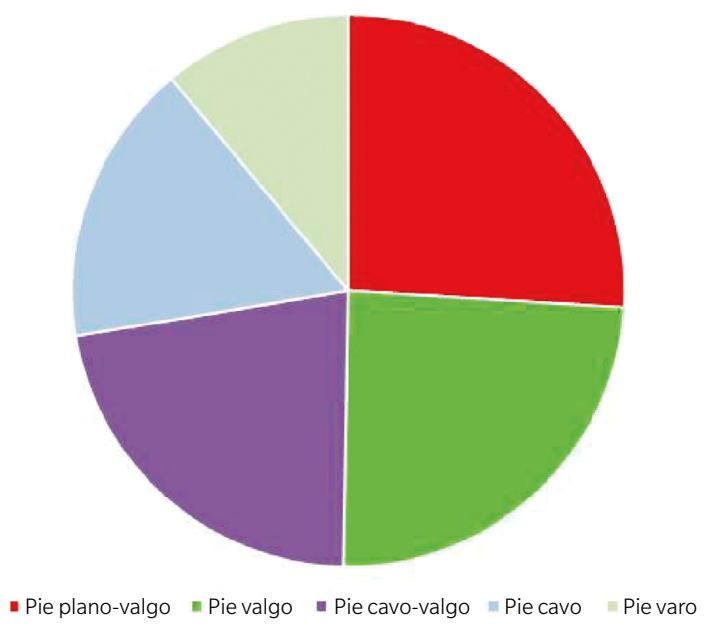

Figura 1. Diagnósticos más frecuentes. 


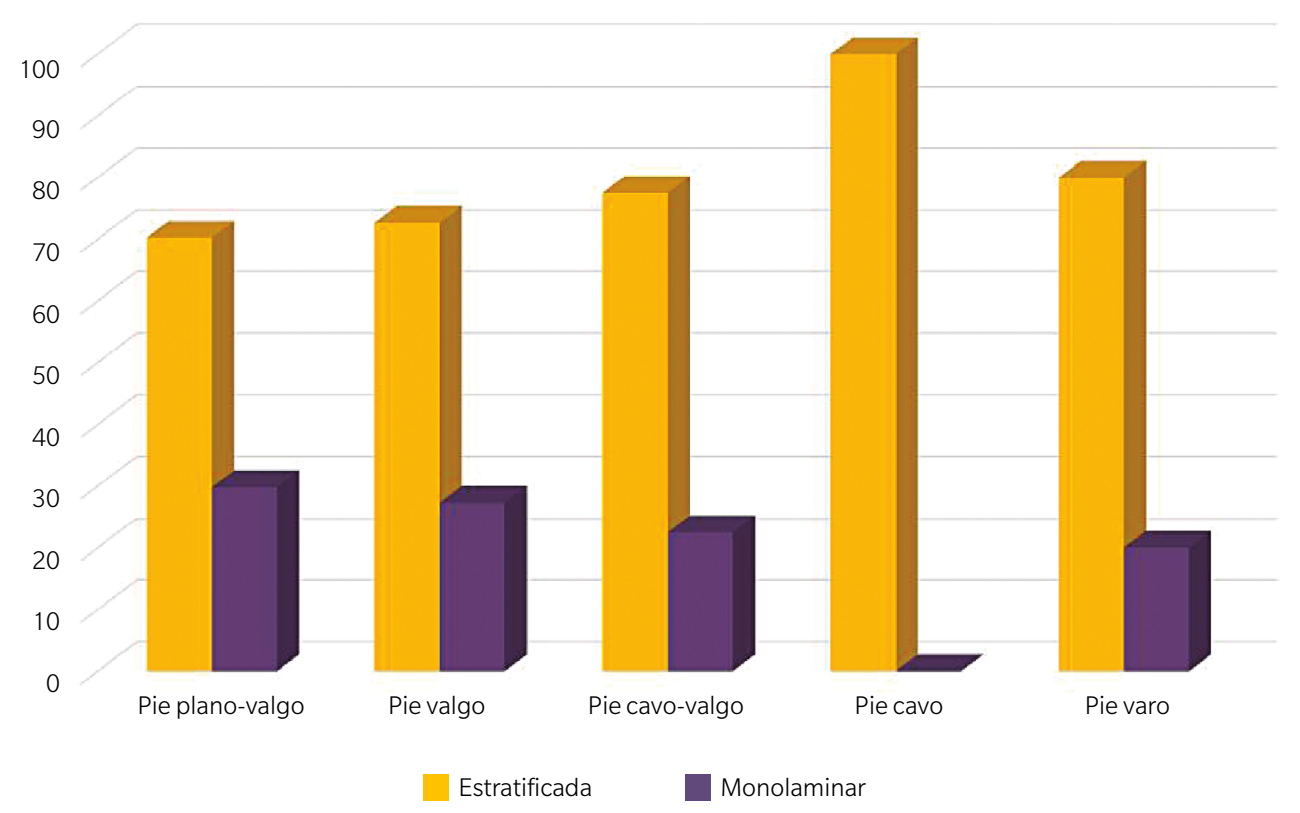

Figura 2. Tipo de ortesis plantares usadas según patología.

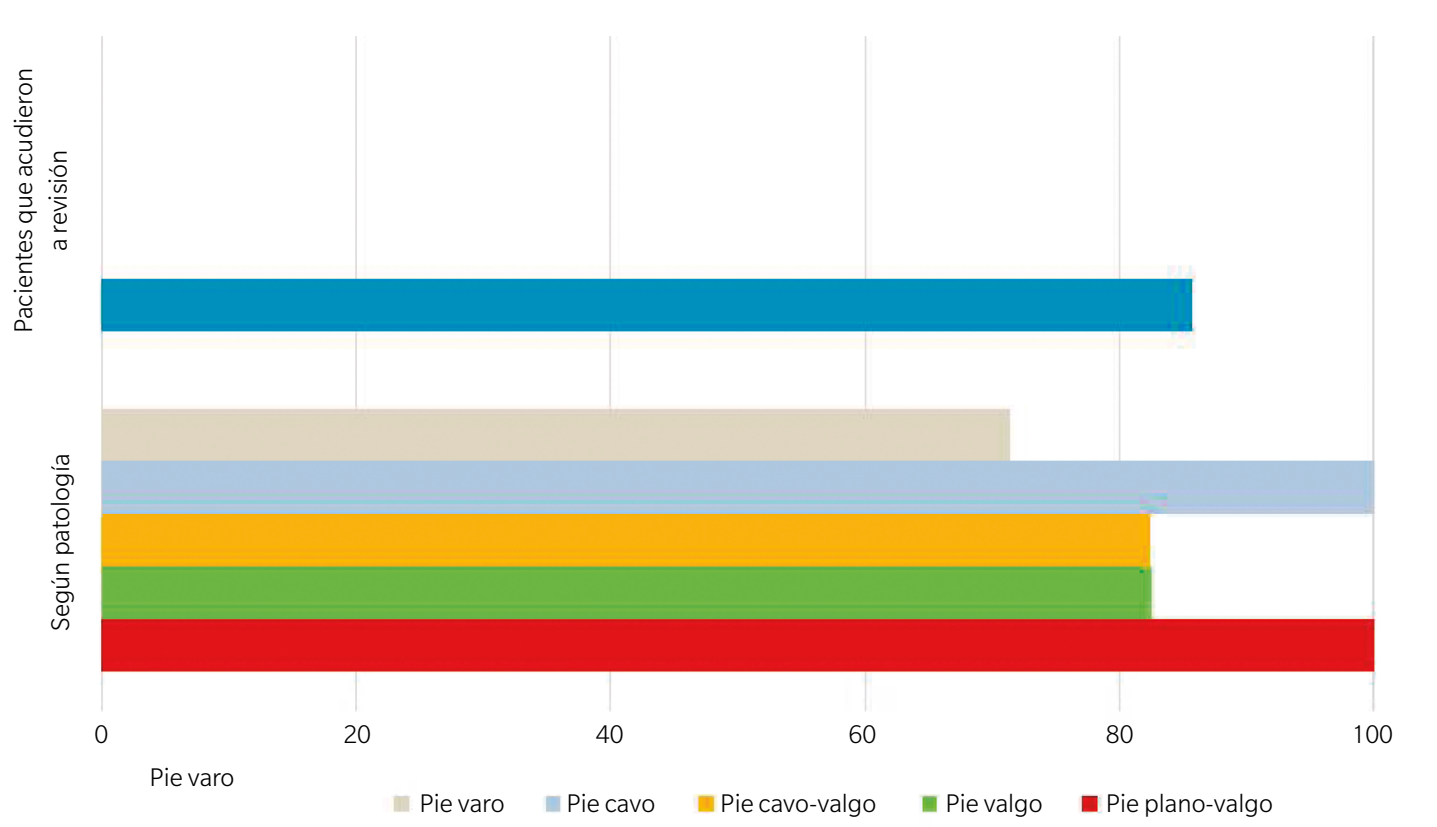

Figura 3. Porcentajes de mejoría después del tratamiento ortopodológico.

El tipo de ortesis plantar que más se prescribió como tratamiento fue la estratificada en el $77.6 \%(n=223)$ de las patologías (Figura 2). Cuando se analizó el tipo de ortesis por edad se observó que la estratificada fue prescrita a sujetos de mayor edad (media $=39.7, \mathrm{DE}=18.9$ ) que la monolaminar (media $=21, \mathrm{DE}=20.4)[\mathrm{T}=-7.3, \mathrm{p}<0.001]$, existiendo asociación entre ser menor de 16 años y ser usuario de una ortesis plantar monolaminar ( $O R=12.6, I C$ $95 \%$ : 6.8-23.3).
Requirió tratamiento complementario el $9.4 \%(n=31)$ de los sujetos, siendo el tratamiento físico el que se prescribió con mayor frecuencia (74.2\%).

Del total de pacientes, tan solo el $46.7 \%(n=154)$ acudió a revisión, comportándose igual por sexo y por edad en esta variable. El 85.7 \% de los pacientes que fueron a revisión presentaron mejoría tras implantar el tratamiento corrector (Figura 3). El tiempo medio para experimentar la mejoría fue de 2.4 semanas $(D E=0.99)$. 
El material más utilizado para la confección del cuerpo de la ortesis en todas estas patologías fue el polipropileno de 3 $\mathrm{mm}$. El elemento de corrección o compensación más usado fue el posteado interno de talón en todos los pies con un componente valgo, y el posteado externo, tanto en el pie cavo como varo.

El elemento de amortiguación más usado fue la cubierta de espuma vinílica EVA, con un grosor de $3 \mathrm{~mm}$ en aquellos pies con un componente valgo, y de $4 \mathrm{~mm}$ en los diagnosticados como pie cavo y pie varo, y las durezas usadas nunca fueron blandas.

Resultados en los pacientes diagnosticados de pie plano-valgo

La ortesis plantar que más se prescribió fue la estratificada en el $70.2 \%(n=33)$ de los casos, requiriendo tratamiento complementario el $4.3 \%(n=2)$ de ellos. El $100 \%$ de los pacientes presentaron mejoría tras implantar el tratamiento ortopodológico.

El material más usado en el refuerzo en las ortesis estratificadas fue el polipropileno en el $87.9 \%(n=29)$ de los casos, usándose resinas en el resto. El grosor más predominante de la cubierta fue de $3 \mathrm{~mm}$ en el $48.5 \%(n=16)$. El $51.6 \%(n=17)$ de estas ortesis llevaba integrado algún otro elemento, siendo el posteado interno de talón el más predominante (30.3\%). La ortesis plantar monolaminar se prescribió al $29.8 \%(n=14)$ de pacientes con este tipo de patología, estando el $64.3 \%$ $(\mathrm{n}=9)$ realizadas de polipropileno de $3 \mathrm{~mm}$. El $64.3 \%$ de los pacientes $(n=9)$ Ilevaba integrada en la ortesis algún otro elemento, siendo el posteado interno de talón el más predominante (50\%). El $28.6 \%(n=4)$ presentaba dos elementos dentro de la plantilla, siendo estos la cuña rotadora externa y el posteado interno de talón en todos los casos. Se observó que la ortesis plantar estratificada fue prescrita a sujetos de mayor edad (media $=30.8, \mathrm{DE}=20.3$ ) que la monolaminar ( media $=12.1, \mathrm{DE}=17.9)[\mathrm{T}=-3.0, \mathrm{p}=0.005]$, existiendo asociación entre ser menor de 16 años y el uso de una ortesis monolaminar (OR = 22.3, IC 95 \%: 4.0-123.7). No se encontraron diferencias en cuanto al tiempo de recuperación entre los dos tipos de ortesis plantar.

\section{Resultados en los pacientes diagnosticados de pie valgo}

El tipo de ortesis plantar que más se prescribió fue la estratificada en el $72.7 \%(n=32)$ de estos, requiriendo tratamiento complementario el $4.5 \%(n=2)$ de los sujetos. El $82.6 \%$ de los pacientes que acudieron a revisión presentaron mejoría tras implantar el tratamiento ortopodológico, sin asociarse con el sexo, la edad o la presencia de dolor previo.

El material más usado en el refuerzo en las ortesis estratificadas fue el polipropileno nuevamente en el $87.9 \%(n=29)$ de los casos, usándose resinas en el resto. El grosor más pre- dominante de la cubierta fue de $3 \mathrm{~mm}$ en el $48.5 \%(n=16)$. El $50 \%(n=16)$ de estas ortesis llevaba integrado algún otro elemento, siendo el posteado interno de talón el más predominante $(18.7 \%)$. El $9.4 \%(n=3)$ presentaban dos elementos de forma conjunta en la ortesis y un paciente presentaba tres elementos de forma simultánea. La ortesis monolaminar se prescribió al $27.3 \%(n=12)$ de los pacientes con este tipo de patología, estando el $58.3 \%(n=7)$ realizadas de polipropileno de $3 \mathrm{~mm}$. El $83.3 \%(n=10)$ de los pacientes llevaba integrado algún elemento, siendo el posteado interno de talón el más predominante (41.7\%). Ninguna presentaba dos elementos de forma conjunta.

Se observó que la ortesis plantar estratificada fue prescrita a sujetos de mayor edad (media $=31.9, \mathrm{DE}=18.5$ ) que la monolaminar (media $=18.0, \mathrm{DE}=21.6)[\mathrm{T}=-2.1, \mathrm{p}=0.04]$, existiendo asociación entre ser menor de 16 años y el uso de una ortesis monolaminar ( $O R=10,29$, IC 95 \%: 2.2-48.7).

\section{Resultados en los pacientes diagnosticados de pie cavo-valgo}

El tipo de ortesis plantar que más se prescribió como tratamiento fue la estratificada en el $77.5 \%(n=31)$ de estos, requiriendo tratamiento complementario el $2.5 \%(n=1)$ de los sujetos. El $82.4 \%$ de los pacientes presentaron mejoría tras implantar el tratamiento ortopodológico, sin asociarse la mejoría con el sexo, la edad o la presencia de dolor previo.

El material más usado en el refuerzo en las ortesis estratificadas fue también el polipropileno en el $87.1 \%(n=27)$ de los casos, usándose resinas en el resto. El grosor más predominante de la cubierta fue de $4 \mathrm{~mm}$ en el $48.4 \%(n=15)$. El $51.6 \%(n=16)$ de estas ortesis llevaba integrado algún otro elemento, siendo una vez más el posteado interno de talón el más predominante (25.8\%). La ortesis monolaminar se prescribió al $22.5 \%(n=9)$ de los pacientes con este tipo de patología, estando el $77.8 \%(n=7)$ realizadas de polipropileno de $3 \mathrm{~mm}$. El $44.4 \%(n=4)$ de los pacientes llevaba integrado algún elemento en la ortesis plantar, siendo la cuña rotadora externa la más predominante (44.4\%). Cuando se analizó el tipo de ortesis plantar por edad no se observaron diferencias significativas entre ellas.

\section{Resultados en los pacientes diagnosticados de pie cavo}

El único tipo de ortesis plantar que se prescribió como tratamiento fue la estratificada en el $100 \%(n=30)$ de estos, requiriendo tratamiento complementario el $13.3 \%(n=4)$ de los sujetos. El $100 \%$ de los pacientes que acudieron a revisión presentaron mejoría tras implantar el tratamiento ortopodológico, sin asociarse la mejoría con el sexo, la edad o la presencia de dolor previo. 
El material más usado en el refuerzo en las ortesis estratificadas fue el polipropileno en el $76,7 \%(n=23)$ de los casos, usándose espuma de polietileno (EPE) en el 6,7\% ( $n=2)$, y resinas en el resto. El grosor más predominante de la cubierta fue de $4 \mathrm{~mm}$ en el 53,3\% $(n=16)$. El $30 \%(n=16)$ de estas ortesis llevaba integrado algún otro elemento, siendo el posteado externo de talón el más predominante (25,8\%).

\section{Resultados en los pacientes diagnosticados de pie varo}

El tipo de ortesis plantar que más se prescribió como tratamiento fue la estratificada en el $80 \%(n=16)$ de estos, requiriendo tratamiento complementario el $5 \%(n=1)$ de los sujetos. El $71.4 \%$ de los pacientes presentaron mejoría tras implantar el tratamiento ortopodológico, sin asociarse la mejoría con el sexo, la edad o la presencia de dolor previo.

El material más usado en el refuerzo en las ortesis estratificadas fueron las resinas en el $43.8 \%(n=7)$ de los casos, usándose polipropileno en el $31.2 \%(n=6)$ y espumas de polietileno en el resto. El grosor más predominante de la cubierta fue de $4 \mathrm{~mm}$ en el $68.8 \%(n=11)$. El $37.5 \%(n=6)$ de estas ortesis llevaba integrado algún otro elemento, siendo el posteado externo de talón el más predominante (25\%). La ortesis monolaminar se prescribió al $20 \%(n=4)$ de los pacientes con este tipo de patología, estando el $75 \%(n=3)$ realizadas de polipropileno de $3 \mathrm{~mm}$. El $75 \%(n=3)$ de los pacientes llevaba integrado algún elemento en la ortesis plantar, siendo siempre el apoyo del primer radio.

\section{DISCUSIÓN}

El objetivo del estudio era conocer qué tipo de tratamiento ortésico ha sido más efectivo en las alteraciones biomecánicas que se diagnosticaron con mayor frecuencia en el Servicio de Ortopodología del ACP de la Universidad de Sevilla, en función del éxito-fracaso de los mismos. La intención final a largo plazo es poder realizar una guía que indique el tratamiento ortopodológico más adecuado a prescribir en estas patologías.

Podríamos resaltar algunas limitaciones del estudio, como por ejemplo que la diversidad de diagnósticos encontrados en las historias revisadas ha hecho que solo podamos obtener resultados significativos de cinco patologías diferentes, correspondientes a las más prevalentes en este servicio de la ACP durante el periodo de tiempo estudiado (años 2013 y 2014). Otra limitación podría ser la elevada falta de asistencia de los pacientes a la revisión posterior programada para comprobar el resultado del tratamiento instaurado, lo cual se ha intentado subsanar mediante encuesta telefónica. Y por último, la falta de precisión, en ocasiones, encontrada en las historias clínicas para describir el tipo de tratamiento y/o los materiales usados en la realización de las ortesis plantares también ha supuesto una limitación para la recogida de los datos, y ha hecho que se tuvieran que descartar un gran número de ellas. Asimismo, información como la dureza, sobre todo, y el grosor del material usado para la cubierta y alargo de las ortesis han quedado incompletos en ciertos casos, por lo que algunas variables han contado con menos datos que otras. Todo ello hace que, a pesar del número de historias revisadas, los resultados no hayan sido tan numerosos y específicos como los esperados. Sin embargo, sí se observa, en las patologías analizadas, la existencia de un patrón de actuación ortopodológico efectivo basado en la instauración de un determinado tipo de ortesis plantar y elementos según el diagnóstico realizado.

Otro de los obstáculos encontrados, y que a su vez fundamenta el fin de este estudio, es la falta de bibliografía existente al respecto. Hawke y cols. ${ }^{3}$ ya comentaron este hecho hace unos años en las conclusiones de su revisión sistemática sobre el tratamiento de la sintomatología dolorosa del pie mediante el uso de ortesis plantares personalizadas. Afirman que producen una mejora importante de la clínica, e incluso de la función del pie en algunos casos, pero que es necesario investigar más para identificar las características que explicarían estos hechos al existir una evidencia científica limitada sobre la que basar las decisiones en cuanto a la prescripción de las ortesis plantares.

Hay varios estudios que se centran en el tratamiento de la sintomatología dolorosa y mejora de la función del pie y, por tanto, mejora de la calidad de vida de los pacientes, según la patología. Varios autores los inician con la convicción de que el uso de una ortesis plantar a medida es la mejor opción para ello, e intentan explicar por qué y cómo. Lee y Hertel ${ }^{4}$, por ejemplo, señalan que la mejora de la alineación de la parte posterior del pie, tanto en un retropié valgo como varo, modifica el reparto de las presiones plantares y previene la aparición de lesiones asociadas al factor de riesgo que suponen la eversión e inversión excesivas del calcáneo. Sin embargo, no hablan de los tipos de ortesis plantares, materiales y elementos que podrían usarse para conseguir esa alineación del pie a la que hacen referencia. Hay autores que afirman que, en los pacientes con un pie plano, el uso de soportes plantares hechos a medida mejoran significativamente la sintomatología y funcionalidad del pie. Khan y cols. ${ }^{5}$ indican que el objetivo mínimo de la ortesis, en este caso, debe ser dar apoyo a la parte medial del pie y controlar el valgo del retropié mediante el uso de una cuña medial de talón. Rosenbloom ${ }^{6}$ expone que una ortesis plantar realizada con una espuma como único material resulta suficiente si el objetivo del tratamiento es solo dar apoyo al pie sin cambiar su estructura, pero que en el caso de querer modificar la biomecánica del paciente, sería necesario corregir la posición del pie para minimizar su eversión mediante elementos como el ángulo de antepié varo y el encapsulamiento medial y lateral del talón, junto con el uso de un calzado adecuado que contenga a la ortesis. También indica, en este último caso, el uso de un refuerzo de polipropileno para controlar y dar apoyo, junto con una cubierta o forro de espuma vinílica (EVA) para aumentar el confort. Asimismo, 
añade la posibilidad de usar un refuerzo en el arco longitudinal interno (ALI) con una EVA para proporcionar un mayor apoyo. Saeedi y cols. ${ }^{7}$ subrayan que, además de aumentar la altura de la articulación subastragalina (ASA), mejorar la alineación del pie y disminuir la sintomatología dolorosa del pie plano-valgo, el uso de un soporte plantar a medida aumenta la actividad del músculo peroneo lateral largo y disminuye la fuerza de reacción del suelo que actúa sobre el pie.

En el caso del pie cavo, Najafi y cols. ${ }^{8}$ sugieren que el uso de un soporte plantar a medida es una solución terapéutica eficaz que puede mejorar significativamente su sintomatología al redistribuir las cargas. Se basan en un estudio realizado por el cual obtienen como resultados la reducción del dolor en hasta un $75 \%$ de los pacientes con este diagnóstico. Burns y cols. ${ }^{9}$ comentan la ausencia de unas pautas claras a seguir en la prescripción del tipo de soporte plantar necesario a usar en esta patología. En su estudio, consiguen reducir totalmente el dolor en un $27 \%$ de los casos, y parcialmente en un $75 \%$, solo mediante el uso de polipropileno de $3 \mathrm{~mm}$ de grosor como refuerzo de la ortesis y una cubierta de Poron, de $3 \mathrm{~mm}$ también, disminuyendo la presión en el antepié y el talón, y aumentando la de la zona medial. Afirman que la clave de un tratamiento exitoso es realizar un soporte con un refuerzo flexible, moldeado según la morfología del pie, y el uso de una cubierta amortiguadora y duradera. LoPiccolo y cols. ${ }^{10}$, especifican con mayor precisión los elementos que debe tener la ortesis en los pacientes con un pie cavo. Hablan del uso de una ortesis plantar de EVA, pero en la que se usarían, para reducir el dolor y la inestabilidad de tobillo, una descarga del primer radio, un ángulo de antepié valgo, un descenso de la altura del ALI (para permitir la eversión del pie) y una talonera. Saeedi y cols. ${ }^{7}$ añaden a lo anteriormente dicho que el uso de un posteado externo de talón reduce la tensión lateral ligamentosa a nivel de la ASA, que el encapsulamiento del talón aumenta la amortiguación natural de la grasa plantar, y que si el primer radio de encuentra plantarflexionado, este debe descargarse con un material amortiguador.

Los resultados de nuestro estudio también demuestran la eficacia del uso de soportes plantares adaptados a la morfología del pie de cada paciente. El uso de una cubierta amortiguadora de EVA, tanto en los pies con un componente cavo y valgo, es igualmente mayoritario, como en el resto de estudios comparados, por lo que podría decirse que es un importante y eficaz elemento amortiguador a usar en las ortesis plantares cuando haya una sintomatología dolorosa por un aumento de la presión plantar. El uso de un material más flexible aumenta en aquellos pies con un componente cavo, coincidiendo con lo que comenta Burns y cols. ${ }^{9}$. El polipropileno de $3 \mathrm{~mm}$ resultó ser el material más versátil como refuerzo, ya que se indicó en la mayoría de las ortesis. Este último autor, al igual que Rosenbloom ${ }^{6}$, usan en sus respectivos estudios el mismo material y grosor para tratar patologías opuestas (pie cavopie plano), obteniendo, de la misma manera que nosotros, resultados positivos. Por último, y debido a la falta de datos, solo podemos confirmar que el uso de un posteado interno de talón, para los pies con un componente plano-valgo, y un posteado externo de talón, para los pies con un componente cavo-varo, son los más usados para el control de la inversión y eversión del pie en su parte posterior respectivamente, y por los resultados obtenidos tanto por nosotros como por los demás autores, unos de los más importantes para mejorar la clínica en los pacientes que presenten un pie con estas características.

Este trabajo podría contribuir en un futuro a la creación de un protocolo de tratamiento ortopodológico de las patologías más prevalentes en el pie. Para ello se necesitaría recopilar el mayor número de datos posibles y ampliar la cantidad de diagnósticos y tipos de tratamiento ortopodológico a usar, además de especificar los materiales, elementos y características estos que deben poseer para conseguir una resolución exitosa de los casos.

Para concluir, hemos encontrado que las patologías diagnosticadas a la mayor parte de los pacientes que acudieron al Servicio de Ortopodología del ACP de la Universidad de Sevilla incluidos en este estudio fueron el pie plano-valgo, el pie valgo, el pie cavo-valgo, el pie cavo y el pie varo. La mejoría producida en los pacientes que acudieron a revisión tras la instauración del tratamiento ortopodológico fue del $71,4 \%$ en los que presentaron un pie varo, mayor del $80 \%$ en aquellos con pie valgo y pie cavo-valgo, y del $100 \%$ en los diagnosticados de pie plano-valgo y pie cavo. El tipo de ortesis plantar prescrita con mayor frecuencia en todas estas patologías fue la estratificada. Con excepción del pie varo, en donde las resinas han sido las más usadas, el polipropileno fue el principal material empleado para la confección del cuerpo de los soportes plantares en el resto de patologías. La cubierta de EVA fue la más utilizada como elemento amortiguador en todas ellas, y los posteados internos y externos de talón los elementos de control-corrección presentes en la mayoría de los casos.

\section{CONFLICTO DE INTERESES}

Los autores declaran no tener conflicto de intereses.

\section{FINANCIACIÓN}

Ninguna.

\section{BIBLIOGRAFÍA}

1. Levy Benasuly A, Cortés Barragán JM. Ortopodología y aparato locomotor. Ortopedia de pie y tobillo. Barcelona: Masson; 2003.

2. MLA. Os Materiais e a sua aplicaçao na Podologia. Vale do Sousa: Escola Superior de Saúde; 2001.

3. Hawke F, Burns J, Radford J, du Toit V. Custom foot orthoses for the treatment of foot pain: a systematic review. J Foot Ankle Res. 2008; 1(Suppl 1):O46. DOI: 10.1186/1757-1146-1-S1-O46. 
4. Lee SY, Hertel J. Effect of static foot alignment on plantar-pressure measures during running. J Sport Rehabil. 2012;21(2):137-43. DOI: 10.1123/jsr.21.2.137.

5. Khan MN, Jacobs BC, Ashbaugh S. Considerations in Footwear and Orthotics. Prim Care - Clin Off Pract. 2013;40(4):1001-12.

6. Rosenbloom KB. Pathology-designed custom molded foot orthoses. Clin Podiatr Med Surg. 2011;28(1):171-87. DOI: 10.1016/j. cpm.2010.11.001.

7. Saeedi H, Mousavi ME, Majddoleslam B, Rahgozar M, Aminian G, Tabatabai Ghomshe F, et al. The evaluation of modified foot orthosis on muscle activity and kinetic in a subject with flexible flat foot:
Single case study. Prosthet Orthot Int. 2014;38(2):160-6. DOI: 10.1177/0309364613492170.

8. Najafi B, Barnica E, Wrobel JS, Burns J. Dynamic plantar loading index: Understanding the benefit of custom foot orthoses for painful pes cavus. J Biomech. 2012;45(9):1705-11. DOI: 10.1016/j.jbiomech.2012.03.006.

9. Burns J, Crosbie J, Ouvrier R, Hunt A. Effective Orthotic Therapy for the. J Am Podiatr Med Assoc. 2006;96(3):205-11. DOI: 10.7547/0960205.

10. LoPiccolo M, Chilvers M, Graham B, Manoli A. Effectiveness of the cavus foot orthosis. J Surg Orthop Adv. 2010;19(3):166-9. 